data. This discussion highlights the issues that need to be resolved and focused on the role of the analytical chemistry in the process.

Table 4. Reliability of the analytical process

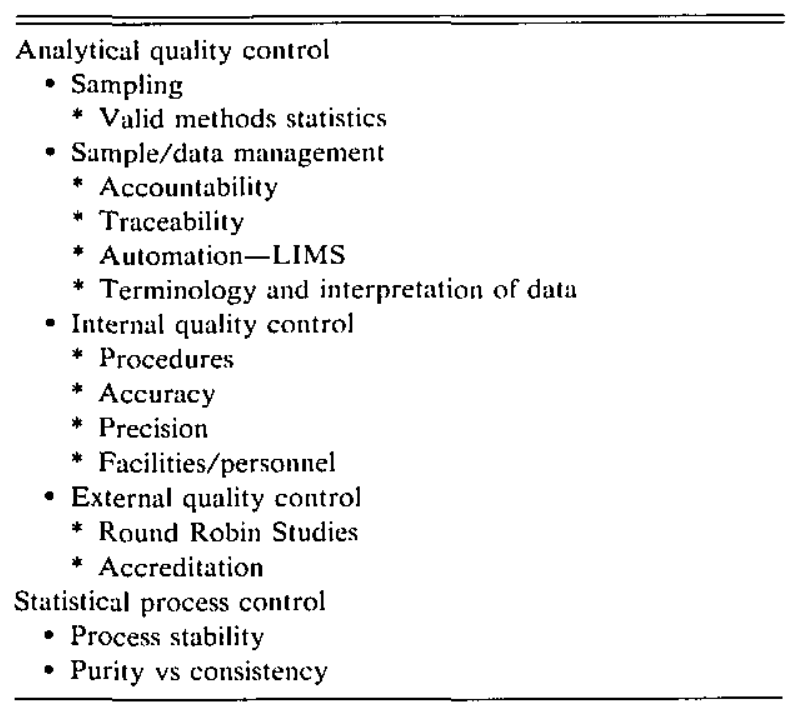

\section{Determination of Traces of Uranium and Thorium in Microelectronics Constituent Materials}

\author{
Hideo Saisho, Masataka Tanaka, \\ and Koichi Nakamura
Toray Research Center, Inc. 2-1, Sonoyama 3-Chome Otsu, Shiga 520, Japan

\section{Eiji Mori}

Sunric Co., Ltd. 2-8-9 Keihinzima, Ota-ku

Tokyo 143, Japan

\section{Introduction}

Because of the problem of soft errors due to $\alpha$-particle emission by uranium and thorium [1], determination of trace amounts of these metals in microelectronics constituent materials has recently gained considerable attention. In the present paper, four different techniques: chemical analysis (CA), instrumental neutron activation analysis (INAA), glow discharge mass spectrometry (GD/MS), and inductively coupled plasma mass spectrometry (ICP/MS) were studied using high-purity aluminum samples $\mathrm{A}, \mathrm{B}$, and $\mathrm{C}$.

\section{Experimental \\ 2.1 Chemical Analysis}

Samples (1-5 g) were decomposed with nitric and hydrochloric acids, evaporated to dryness, dissolved in nitric acid and subjected to chemical analysis.

2.1.1 Uranium Analysis [2,3] With sodium nitrate and aluminum nitrate added into nitric acid solutions of samples, uranium was extracted twice with a carbon tetrachloride solution of tributyl phosphate (TBP). The organic phase was backextracted thrice with dilute hydrochloric acid after being washed with a sodium nitrate solution and hydrochloric acid. The aqueous phase was evaporated to dryness. The residue was fused with the addition of a fusing mixture consisting of potassium carbonate, sodium carbonate and sodium fluoride. The fused product was set in a fluorophotometer to determine the uranium by measuring the fluorescence intensity at $556 \mathrm{~nm}$.

2.1.2 Thorium Analysis [4,5] Aluminum nitrate and EDTA were added to nitric acid solutions of samples and the resulting solutions were extracted twice with mesityl oxide. The organic phase was back-extracted thrice with water. The aqueous phase, with acids added, was decomposed by heating. The residue was dissolved in hydrochloric acid and with Arsenazo III added to this solution, thorium was determined by measuring the absorbance at $660 \mathrm{~nm}$.

\subsection{INAA}

About $5 \mathrm{~g}$ of each sample was irradiated for $24 \mathrm{~h}$ at a thermal neutron flux of $5.5 \times 10^{11}$ neutrons $\mathrm{cm}^{-2} \mathrm{~s}^{-1}$. After cooling, the irradiated sample was counted for $10,000 \mathrm{~s}$ using a $\mathrm{Ge}(\mathrm{Li})$ detector. The cooling time was 6 to 8 days for uranium and 15 to 19 days for thorium. Uranium was determined by measuring $\gamma$-rays from $228 \mathrm{keV}$ of ${ }^{2.39} \mathrm{~Np}$ and thorium from $312 \mathrm{keV}$ of ${ }^{2.33} \mathrm{~Pa}$.

\subsection{GD/MS}

The instrument used was a commercial GD/MS system (VG 9000, VG Isotopes). A pin-shaped 


\section{Accuracy in Trace Analysis}

sample was prepared for measurement. Discharge was conducted, using argon as the discharge gas, with a voltage of $1.0 \mathrm{kV}$ and current of $2.0 \mathrm{~mA}$. Quantitative analysis was performed at a mass resolution of 5400 with a data acquisition time of 75 to $150 \mathrm{~s}$ per isotope.

\section{$2.4 \mathrm{ICP} / \mathrm{MS}$}

Using a PlasmaQuad (VG Isotopes), measurements were taken with two kinds of sample solutions: one was for acid-decomposition, and the other for chemical separation of uranium and thorium from an acid-decomposed solution as in the case of chemical analysis.

In mass spectrometry, the isotopes of uranium and thorium measured were ${ }^{238} \mathrm{U}$ and ${ }^{232} \mathrm{Th}$, respectively.

\section{Results and Discussion}

\subsection{Accuracy and Precision}

The accuracy and precision of the chemical analysis were confirmed using NBS reference materials. As can be seen from tables 1 and 2, our chemical analysis methods were found to have acceptable accuracy and precision.

Table 1. Accuracy data for NBS reference materials

\begin{tabular}{|c|c|c|c|c|}
\hline \multicolumn{5}{|c|}{ This work (ppm) } \\
\hline & Run 1 & Run 2 & Mean & Certified value (ppm) \\
\hline 1. Uranium & & & & \\
\hline $\begin{array}{l}\text { SRM } 616 \\
\text { (glass wafer) }\end{array}$ & 0.068 & 0.069 & 0.068 & $0.0721 \pm 0.0013$ \\
\hline 2. Thorium & & & & \\
\hline $\begin{array}{c}\text { SRM } 614 \\
\text { (glass wafer) }\end{array}$ & 0.75 & 0.74 & 0.74 & $0.748 \pm 0.006$ \\
\hline
\end{tabular}

Table 2. Precision data for the NBS 688 sample

\begin{tabular}{lcc}
\hline & Uranium (ppm) & Thorium (ppm) \\
\hline Run 1 & 0.31 & 0.31 \\
Run 2 & 0.33 & 0.31 \\
Run 3 & 0.28 & 0.32 \\
Run 4 & 0.30 & 0.30 \\
Run 5 & 0.32 & 0.30 \\
Mean & 0.31 & 0.31 \\
CV" & $6 \%$ & $3 \%$ \\
\hline
\end{tabular}

SRM 688 (basalt rock): U. $0.37 \mathrm{ppm}$ (non-certified).

Th, $0.33 \pm 0.02 \mathrm{ppm}$ (certified).

${ }^{\mathrm{h}}$ Coefficient of variation [(standard deviation/mean $\left.)(100)\right]$

\subsection{Comparison of Results Obtained by Four Analytical Methods}

Table 3 summarizes results obtained by chemical analysis, INAA, GD/MS and ICP/MS. So far as ICP/MS is concerned, results obtained by direct measurements of acid-decomposition solutions are omitted, because of unreliable nature of these results when the concentrations are lower than $100 \mathrm{ppb}$.

Table 3. Analytical results for high-purity aluminum samples obtained by four different methods

\begin{tabular}{|c|c|c|c|c|c|c|c|}
\hline & & \multicolumn{2}{|c|}{ Sample A } & \multicolumn{2}{|c|}{ Sample B } & \multicolumn{2}{|c|}{ Sample C } \\
\hline & & $\mathrm{U}$ & $\mathrm{Th}$ & $\mathrm{U}$ & Th & $\mathrm{U}$ & Th \\
\hline $\mathrm{CA}$ & (ppm) & 0.001 & $<0.01$ & 0.010 & 0.02 & 0.080 & 0.07 \\
\hline INAA & (ppb) & $<1$ & $1.6 \pm 0.8$ & $10 \pm 2$ & $14 \pm 2$ & $82 \pm 4$ & $79 \pm 4$ \\
\hline $\mathrm{GD} / \mathrm{MS}$ & $(p p b)^{a}$ & $<5$ & $<5$ & $15 \pm 3$ & $18 \pm 2$ & $121 \pm 17$ & $119 \pm 8$ \\
\hline $\mathrm{ICP} / \mathrm{MS}^{\mathrm{h}}$ & $(\mathrm{ppb})$ & 2.2 & 5.3 & 12 & 12 & 79 & 84 \\
\hline
\end{tabular}

"Ion beam ratio. Data not corrected using RSF. Mean \pm standard deviation (5 determinations).

"Data obtained using the same solvent extraction procedure for CA.

Three samples subjected to chemical analysis and INAA provide close agreement. Results of measurements by ICP/MS with Samples B and C are in close agreement with those by chemical analysis and INAA. However, with Sample A with the lowest concentrations of both uranium and thorium, somewhat higher analytical values have been obtained in ICP/MS than in INAA. The spectral signals observed in ICP/MS from Sample A's uranium and thorium were apparently different from those of the blanks. On these grounds, the analytical values seem to have high accuracy.

The measured values by GD/MS are given in terms of ion beam ratios relative to that of an aluminum matrix. They are higher than the values obtained by the other three analytical methods with both Samples B and C. However, the ratio of the mean value of GD/MS to that of INAA falls in a range of 1.3-1.5 with both elements. Thus, by correction using this ratio or a relative sensitivity factor (RSF), satisfactory agreement can be obtained between the results from GD/MS analysis and the other three different analyses. If a more accurate RSF is obtained, it will become possible to make determinations of uranium and thorium very rapidly at concentration levels of $10 \mathrm{ppb}$ or higher, as compared with the other three analytical methods. 


\section{Acknowledgment}

The authors are grateful to Marubun Corporation (Tokyo, Japan) fo" the ICP/MS measurements.

\section{References}

[1] May, T. C., and Woods, M. H., 16th Proceedings of 1978 International Reliability Physics Symposium, p. 33. San Diego, CA, April 1978.

[2] Science and Technology Agency, Methods for Determiniltion of Uranium, Japan Chemical Analysis Center. Chiba (1982) p. 9.

[3] Sandell, E. B., Colorimetric Determination of Traces of Metals, Interscience Publishers, Inc., New York (1959) p. 908.

[4] Grimaldi, F. S., Treatise on Analytical Chemistry. Part II, 5, Interscience Publishers, Inc., New York (1961) p. 139.

[5] Trofimova, L. A., and Syromyatnikov, N. G., Determination of Uranium, Thorium, and Zirconium with Arsenazo III without Chemical Separation, Zavodsk. Lab. (U.S.S.R.) 31, 1325 (1965); C.A. (U.S.) 64, 4254 (1966).

\section{Multitechnique Approach to Trace Characterization of High Purity Materials: Gallium}

\author{
S. Gangadharan, S. Natarajan, \\ J. Arunachalam, S. Jaikumar, and \\ S. V. Burangey \\ Ultratrace and Nuclear Methods Section \\ Analytical Chemistry Division \\ Bhabha Atomic Research Centre \\ Trombay, Bombay 400085 India
}

\section{Introduction}

Compositional characteristics complement the structural aspects and in combination with certain correlated property measurements, provide complete characterization of materials. While characteristics completely govern the properties, the properties do not give a unique or complete account of the characteristics. The ultimate constraint in the characterization of ultrapure materials is the ability to maintain the integrity of the sample throughout the analytical measurement process.
Developments in clean-room technology, coupled with the advances in plastics industry, have helped to push down the (environment and reagent) blank levels which has led to increased reliability in the quantitative measurement of impurities at ppb and sub-ppb levels of concentration. The stringent requirement of purity of materials for high technology demand compositional characterization for several (metallic) impurities which necessitates the use of diverse techniques like GFAAS, ICP/AES, activation analysis and electrochemical techniques, the choice of a given technique being guided by the sensitivity and selectivity it offers towards the characterization. To ensure good precision for measurements at ultratrace levels, the analytical methodologies should ensure minimum process blanks and afford (simultaneous) analysis of several critical elements related to (i) the manufacturing process, (ii) chemical similarity with reference to the matrix, and (iii) in the case of semiconductor materials, the donor, acceptor and neutral impurities.

\subsection{The Facility}

A (prototype) ultratrace analytical facility has been set up using indigenously available technology, to establish the attainable levels of cleanliness. The laboratory, designed to be at different levels of cleanliness, is better than class 100 at the work surface. The cleanliness with respect to (metallic) impurities has been monitored through the analysis of the air samples at several locations in addition to particulate counting. Distilled water, passed through a mixed bed ion exchange resin, is being routinely used. The levels of some of the impurities in that water, which reflect the cleanliness of the laboratory environment is as follows:

$\begin{array}{llllll}\text { Element } & \mathrm{Cd} & \mathrm{Cu} & \mathrm{Fe} & \mathrm{Pb} & \mathrm{Zn} \\ \text { Level ng/L } & 3 & 10 & 60 & 10 & 100\end{array}$

These levels have been maintained over several months of operation.

\section{Approaches: Experimental}

The multitechnique approach to trace characterization of gallium metal has been adopted. The philosophy behind the approach has been (i) to ensure cross-validation, i.e., generation of analytical results by at least two independent analytical methodologies wherever possible and obtain complementary information, (ii) to develop optimum instrumental 